\title{
As dimensões sociais dos gêneros musicais: porque os sistemas de classificação comercial e não comercial variam
}

\author{
The social dimension of musical genres: \\ why commercial and non-commercial \\ classification systems vary
}

Rose Marie SANTINI'

\section{Resumo}

O objetivo do artigo é analisar a construção colaborativa de sistemas de classificação no campo da música popular e discutir de que forma podem diferir daqueles enunciados e orientados pela indústria da música até então. Portanto, comparou-se a classificação colaborativa da música popular que emerge na Internet com aquela utilizada pela Indústria Fonográfica, a partir de dados coletados em dois websites: Last.fm e AllMusic. A partir dessa comparação, verificou-se as diferenças existentes entre os critérios de classificação utilizados pelos usuários e os princípios comerciais de classificação encontrados nos catálogos das gravadoras e editores musicais. Os resultados encontrados apontam para uma discrepância entre os critérios utilizados pela Indústria para classificar artistas pertencentes a um mesmo gênero musical e/ou segmento de mercado e aqueles associados pelos usuários e realmente percebidos, apreciados e consumidos por um mesmo público.

Palavras-chave: Classificação colaborativa. Indústria fonográfica. Música popular. Sistemas de classificação.

\begin{abstract}
The aim of the paper was to analyze the collaborative development of classification systems in the field of popular music and discuss how they may differ from those established and guided by the music industry so far. Therefore, we compare the collaborative classification of music that emerges on the Internet with that used by the Phonographic Industry based on the data collected at two websites: Last.fm and AllMusic. Through this comparison we found differences between the classification criteria used by users and the commercial principles of classification found in the catalogues of record labels and music publishers. The results indicate adiscrepancy between the criteria used by the industry to classify artists who belong to the same musical genre and/or market segment, and those associated by the users and actually perceived, appreciated and consumed by this public.
\end{abstract}

Keywords: Collaborative classification. Phonographic industry. Popular music. Classification systems.

\section{Introdução}

Uma série de mudanças no âmbito das tecnologias, da economia e das práticas sociais caracteriza a emergência de novas formas de organização social ancoradas em redes de colaboração articuladas por meio da Internet. Esse deslocamento tem permitido o crescimento do papel da produção e da mediação não

\footnotetext{
1 Professora Doutora, Universidade Federal do Rio de Janeiro, Escola de Comunicação, Curso de Comunicação Social. Av. Pasteur, 250, Urca, 22290-902, Rio de Janeiro, RJ, Brasil. E-mail:<marie.santini@eco.ufrj.br>.

Recebido em 25/10/2012, reapresentado em 8/1/2013 e aceito para publicação 17/1/2013.
} 
comercial nos setores da informação e da cultura organizados em um padrão radicalmente mais descentralizado do que aqueles encontrados nos mesmos setores baseados no modelo industrial do século XX. Esse fenômeno vem reforçando a relevância social da produção e do compartilhamento coletivo de informação paralelamente à existência da propriedade, da produção e da mediação baseada no mercado.

Tais transformações ocorrem pelos esforços individuais e coletivos em uma ampla variedade de tipos de colaboração, desde as mais sutis até as mais firmemente tecidas. A emergência dessas práticas sociais apresenta sucessos notáveis em diversas áreas, dentre as quais se destacou a criação de folksonomias e de filtragens colaborativas de informação enquanto processos de classificação de conteúdos.

A classificação colaborativa apresenta-se como um processo inovador de mediação social produzido pelos próprios usuários dos produtos culturais. No caso da música utilizada como campo de investigação neste artigo, a classificação colaborativa funciona como processo de mediação entre os ouvintes e a imensa quantidade de arquivos musicais disponíveis online.

Devido aos desafios subjacentes à organização da informação no domínio da música popular, a classificação dominante desde meados do século XX vem sendo operada pela Indústria Musical que categoriza os produtos culturais de acordo com suas lógicas comerciais. Com o surgimento do fenômeno da folksonomia no entorno colaborativo da Internet, começam a surgir condições no nível da organização social que criam possibilidades de escapar das classificações da música produzida e reproduzida tradicionalmente pelas Indústrias Culturais.

Diante desse contexto, este trabalho pretende verificar o tipo de relação que pode se estabelecer entre a construção colaborativa de um sistema de classificação artístico (no caso, da música) com os padrões de organização social que fazem emergir novos usos sociais da cultura e de que forma tais padrões podem diferir daqueles enunciados e orientados pelas Indústrias Culturais até então. dos usos sociais da música por meio de sua classificação. Por outro e ao mesmo tempo, os rituais de classificação como reveladores dos usos e valores que a música adquire de forma dinâmica e mutável no campo social.

A partir de uma análise empírica, foi proposta a comparação entre a classificação de artistas por categorias baseadas em similaridade, que correspondem a gêneros musicais, em dois domínios: um sistema de classificação comercial amplamente utilizado no mercado musical e outro não comercial criado pelos usuários da Internet. Por meio dessa comparação pretende-se verificar as diferenças existentes entre os critérios de classificação utilizados pelos usuários e os princípios comerciais de classificação encontrados nos catálogos das gravadoras e dos editores musicais. O objetivo é identificar o significado tanto da produção, da reprodução, como das variações entre as categorias nos dois domínios estudados.

A classificação da música em plataformas colaborativas na Internet define a visibilidade das obras em situações de busca e de recuperação de informação. Também para a Indústria da Música a classificação por meio de gêneros pretende promover o encontro entre os produtos e seus públicos, identificando perfis de consumo, nichos, audiências, mercados, contextos comerciais para divulgação e venda de conteúdos. Em ambos os casos, os padrões de classificação da oferta musical organizam e orientam a articulação entre a produção artística, e a conformação da demanda e a criação de novos usos sociais para os produtos culturais e suas dinâmicas devem ser investigadas.

\section{A importância do estudo dos gêneros musicais para o campo cultural}

Na tentativa de evitar os riscos que implicam a observação dos padrões de produção, distribuição e consumo separadamente, optou-se por desenvolver um estudo sobre os usos sociais da música a partir da análise dos sistemas de classificação dos gêneros musicais. A justificativa para a escolha desta abordagem se divide em três argumentos principais, os quais são inspirados em DiMaggio (1987).

Primeiramente, ao investigar os padrões de consumo isoladamente, não se conseguiu explicar por que os usuários fazem determinadas escolhas dentro de um 
amplo repertório e uma ampla variedade da oferta musical. Ou seja, não cabe aqui considerar as categorias artísticas como dadas ou tratar o gosto como algo exógeno aos sujeitos.

Com isso, ao estudar os sistemas de produção sem articular conjuntamente uma teoria sobre a demanda, corre-se o risco de sugerir que a produção e a distribuição dos bens culturais podem ser compreendidas simplesmente como uma imposição aos consumidores. E os produtos que escapam a essa lógica, por exemplo: os fracassos da Indústria, permanecem sem explicação.

Por último, o foco na dinâmica da classificação da música pode ajudar a entender questões como a criação de novos usos sociais; o grau em que os produtos artísticos "refletem" seu ambiente social; a relação entre os usos e a emergência de gêneros na medida em que a classificação é um processo de mediação entre produtores, distribuidores e usuários.

A ambígua noção de gênero musical é central para a discussão. Neste artigo, usou-se a palavra gênero musical para referir-se a um conjunto de obras musicais classificadas conjuntamente com base nas similaridades percebidas tanto pelos usuários como pelo mercado da música. A comparação e identificação das diferenças entre os princípios de classificação dos ouvintes com os da Indústria Musical refletem tanto a estrutura dos gostos dos usuários como a estrutura da produção e distribuição de bens culturais.

Os gêneros representam princípios construídos de organização social que impregnam as obras de significância a partir de seu conteúdo temático, mas também por suas utilidades e usos contextuais. Ou seja, os processos pelos quais as distinções entre os gêneros são criadas, ritualizadas e erodidas e os processos pelos quais os gostos são produzidos fazem parte da construção coletiva dos sentidos das obras que contribui para a delimitação de fronteiras entre os grupos sociais.

Essa perspectiva é especialmente importante quando se considera o objeto de estudo e o contexto de análise como os processos de classificação colaborativa cujos critérios são definidos pelos próprios usuários. A partir desta recente conjuntura de colaboração em rede, o que se quer é identificar as consequências da criação coletiva de um sistema de classificação da música para o campo cultural.

\section{Métodos}

O estudo da classificação da música neste trabalho aplica o método de análise comparativa com o propósito de confrontar a classificação colaborativa da música popular criada pelos usuários da Internet com aquela utilizada pela Indústria Musical. Em ambos os domínios, tais critérios de classificação definem as semelhanças e diferenças entre artistas baseando-se em categorias de gêneros e subgêneros musicais para gerar recomendações aos usuários.

A análise dos domínios foi realizada a partir dos dados coletados em dois websites: Last.fm e AllMusic. Esse método comparativo foi considerado o mais adequado para alcançar os objetivos de pesquisa em função da riqueza dos dados disponíveis e por seu caráter não obstrutivo.

\section{AllMusic}

O AllMusic foi selecionado para representar o sistema de classificação da música popular construído pela Indústria Musical para fins comerciais. O website foi inaugurado em 1995 pelo grupo empresarial All Music Guide (AMG) que atualmente pertence à empresa norte-americana Macrovision Corporation.

O vocabulário e os critérios de classificação da música popular criados e divulgados pelo grupo AllMusic.com são utilizados internacionalmente como referência para a classificação dos catálogos dos produtos culturais na esfera da Indústria Musical. O Theasurus do AllMusic é referência para a organização dos catálogos das grandes gravadoras (EMI, Sony/BMG, Universal e Warner) e é utilizado pelas publicações e consultorias internacionais no campo da música (como a revista Billboard e a consultoria Nielsen Business Solution) e por empresas que atuam na distribuição e venda de música online, como a Microsoft, AOL, Yahoo!, Amazon, Barnes \& Noble, Best Buy, Ticketmaster, Musicmatch, iTunes, Napster, entre outras (Thompson, 2008).

A classificação do material musical no AllMusic conta com o trabalho de um staff editorial permanente composto por especialistas e críticos de música para a organização de um catálogo de um milhão de artistas e mais de 13 milhões de canções. O AllMusic se define como 
"A fonte de referência musical mais compreensível do planeta" (Allmusic, 2009, online). As informações sobre as obras musicais, os compositores, interpretes etc. estão disponíveis gratuitamente na Internet para consulta pelo público interessado.

\section{Last.fm}

Escolheu-se a Last.fm, principal Sistema de Recomendação disponível atualmente na Rede, para representar a classificação realizada pelos usuários da Internet devido à sua popularidade entre os internautas, à sua complexidade tecnológica e ao tamanho de seu acervo. O sistema de recomendação da Last.fm articula inovações tecnológicas que caracterizam sua plataforma online como uma rede social, cuja dinâmica de recomendação é baseada na folksonomia, isto é, por meio de classificação colaborativa do material musical realizada por seus usuários.

Em relação à popularidade, a Last.fm posiciona-se atualmente como a principal rede social de música na Internet, conectando cerca de 37 milhões de usuários diretos e 19 milhões de usuários indiretos - distribuídos em 280 países -, a um acervo de mais de 150 milhões de canções produzidas por 280 mil gravadoras e mais de 16 milhões de artistas independentes (Miller et al., 2008). Além disso, a Last.fm apresenta"A maior classificação ontológica do mundo, o maior catálogo legalmente licenciado para escuta através de streming na Rede e a mais extensa base de dados de música conhecida até o momento" (Henning; Reicheld, 2008, p.1).

\section{Processo de coleta de dados}

Ambos os sites, a Last.fm e o AllMusic, indicam ou recomendam para cada artista selecionado outros artistas similares ou parecidos. A lista de artistas considerados "parecidos" na Last.fm, motor de seu sistema de recomendação, é baseada em dois métodos. O primeiro critério é o hábito musical dos ouvintes. Se muitos usuários ouvem o artista $X$, mas também $Y$ e Z, os artistas $Y$ e $Z$ são identificados como similares a $X$. Uma segunda função é adicionada à equação de similaridade para torná-la mais precisa no sistema de recuperação da Last.fm, baseada nas estatísticas de uso das tags pelos usuários para classificar artistas distintos. Por exemplo, se duas cantoras são classificadas frequentemente pelos usuários com as mesmas tags - como bossa nova ou jazz por exemplo -, são consideradas similares. O sistema cruza as informações coletadas a partir desses dois métodos para indicar os artistas parecidos (Last.fm, 2009).

O site AllMusic indica artistas similares em três categorias: artistas parecidos, seguidores e influências. Conforme mencionado, tal similaridade é definida pelo staff editorial e por críticos de música contratados pelo AllMusic (AllMusic, 2009). Portanto, na Last.fm, a semelhança entre artistas é baseada nos usos dos ouvintes, e, no AllMusic, é baseada na organização e classificação definida por especialistas contratados pela Indústria.

A partir do método de análise comparativa de domínios, buscou-se analisar se os artistas parecidos indicados pelo AllMusic estão presentes nas listas de similaridades indicadas pela Last.fm. O objetivo é verificar se os critérios de classificação utilizados pela Indústria correspondem às percepções, aos usos e gostos dos usuários.

Para a coleta dos dados, foram selecionados 11 tags, dentre as mais populares na Last.fm, que correspondem a um gênero musical estabilizado pela Indústria da Música. Portanto, cada um dos 11 gêneros escolhidos representa um segmento distinto do mercado da música popular mundial, a saber: Rock, Folk, Pop, Electronic (Eletrônico), Country, Hip-Hop, Rhythm and Blues (R\&B), Jazz, Blues, Hardcore, Latin (Latino). Para cada uma dessas 11 tags/gêneros musicais, separou-se o artista mais escutado na Last.fm no mês de março de 2009 (Tabela 1).

Considerou-se esses 11 artistas mais escutados por gênero a fim de se comparar a recomendação da Last.fm com a recomendação do AllMusic. Colocou-se os artistas selecionados em diferentes tabelas de Excel, as quais foram numeradas de Planilhas 1 a 12. Na coluna direita foram inseridos os artistas similares a cada um deles conforme o site da Last.fm. A coluna esquerda foi preenchida com os artistas parecidos indicados pelo AllMusic. Em seguida, analisou-se a porcentagem de 
Tabela 1. Artistas mais populares por gênero na Last.fm.

\begin{tabular}{|c|c|c|c|}
\hline Genêro/Tag & Artista mais popular & Número de ouvintes/mês & Número de execuções/mês \\
\hline Rock & Coldplay & 2162574 & 105313971 \\
\hline Folk & Bob Dylan & 1194990 & 48898452 \\
\hline Pop & Madonna & 1185293 & 36849612 \\
\hline Electronic & Depeche Mode & 1155188 & 43290636 \\
\hline Country & Johnny Cash & 1050251 & 34419422 \\
\hline Hip-Hop & Kanye West & 986279 & 39072857 \\
\hline Rhythm and Blues (R\&B) & Rihanna & 953443 & 21596786 \\
\hline Jazz & Norah Jones & 875199 & 21866624 \\
\hline Blues & Tom Waits & 600978 & 27033292 \\
\hline Hardcore & Rise Against & 554635 & 38242584 \\
\hline Latin & Manu Chao & 535291 & 15757151 \\
\hline
\end{tabular}

Fonte: LAST.FM (2009).

Tabela 2. Compatibilidade entre as recomendações da Last.fm e do AllMusic.

\begin{tabular}{|c|c|c|c|c|c|c|c|}
\hline Gênero & $\begin{array}{c}\text { Artista semelhantes } \\
\text { unicamente na Last.fm (1) }\end{array}$ & $\begin{array}{l}\text { Artista em } \\
\text { comum (c) }\end{array}$ & $\begin{array}{c}\text { Artista semelhantes } \\
\text { unicamente no AllMusic (2) }\end{array}$ & Total & $\% 1^{\prime}$ & $\% C$ & $\% 2^{\prime}$ \\
\hline Country & 54 & 18 & 28 & 100 & 54,0 & 18,0 & 28,0 \\
\hline Rhythm and Blues (R\&B) & 81 & 14 & 8 & 103 & 78,6 & 13,5 & 7,8 \\
\hline Jazz & 53 & 11 & 20 & 84 & 63,1 & 13,1 & 23,8 \\
\hline Blues & 6 & 5 & 35 & 46 & 13,0 & 10,9 & 76,1 \\
\hline Hip-Hop & 81 & 11 & 12 & 104 & 77,9 & 10,6 & 11,5 \\
\hline Latin & 9 & 2 & 8 & 19 & 47,4 & 10,5 & 42,1 \\
\hline Rock & 45 & 9 & 34 & 88 & 51,1 & 10,2 & 38,6 \\
\hline Hardcore & 74 & 10 & 15 & 99 & 74,7 & 10,1 & 15,2 \\
\hline Pop & 33 & 7 & 37 & 77 & 42,9 & 9,1 & 48,1 \\
\hline Folk & 5 & 4 & 45 & 54 & 9,3 & 7,4 & 83,3 \\
\hline Electronic & 6 & 1 & 41 & 48 & 12,5 & 2,1 & 85,4 \\
\hline Total & 447 & 92 & 283 & 822 & 47,7 & 10,5 & 41,8 \\
\hline
\end{tabular}

compatibilidade de artistas parecidos nos dois domínios para cada artista selecionado por gênero musical.

\section{Critérios de análise}

A Last.fm apresenta para cada artista outros 200 artistas parecidos e indica o grau de similitude entre eles em 5 níveis: "super", "muito alta", "alta", "média" e "baixa". Considerou-se apenas os 3 primeiros níveis de similaridade ("super", "muito alta" e "alta") com a finalidade de analisar somente as semelhanças consideradas pela grande maioria dos usuários.

No AllMusic considerou-se as três categorias de artistas similares (artistas parecidos, seguidores e influências) para ampliar ao máximo o espectro de análise de semelhança considerado pelos críticos e expertos do site.

\section{Resultados}

A comparação entre os artistas indicados como similares pelo Allmusic com aqueles indicados pelo sistema Last.fm apresenta forte discrepância, conforme indica a Tabela 2, organizada em ordem decrescente: do gênero que apresenta maior porcentagem de artistas em comum ao de menor compatibilidade.

Dentre os 822 artistas analisados, apenas 92 artistas são semelhantes em ambos os domínios (10,5\%), tanto na Last.fm como no AllMusic, conforme indica a última linha da Tabela 2. No total, 447 artistas aparecem como parecidos unicamente na Last.fm e 283 artistas são indicados como similares somente no AllMusic. Ou seja, em geral, os artistas que a Indústria considera como parecidos, pertencentes ao um mesmo gênero musical, nicho 
e/ou segmento de mercado diferem significativamente daqueles associados pelos usuários e realmente percebidos, apreciados e consumidos por um mesmo público.

\section{Discussão}

Os resultados encontrados nos conduzem a elaboração da seguinte proposição: quanto maior a penetração e o tamanho do mercado de determinado gênero, mais fortes são as influências da classificação comercial no nível da oferta e da demanda. Os argumentos que reforçam tal hipótese baseiam-se na análise da Tabela 2.

No topo da Tabela 2, encontrou-se o gênero "country" seguido do gênero R\&B. O gênero "country"junto com o R\&B, "alternative" e "rap" corresponderam a 50\% das vendas de disco da Indústria Fonográfica em 2008 (Richard, 2009). Considerando que não se analisou nesta pesquisa os dois últimos - "alternative" e "rap" -, percebe-se que os dois gêneros mais vendidos são também os que apresentam maior compatibilidade entre os "arranjos de artistas parecidos" indicados pelos usuários e apontados pela Indústria.

Na extremidade inferior da Tabela 2, encontrou-se o gênero "electronic"(eletrônico). No mercado fonográfico, somadas as vendas de discos físicos e de canções digitais, a música eletrônica corresponde ao gênero de menor rentabilidade comercial, cuja participação representa apenas 3\% do mercado musical. O gênero "electronic" está entre os segmentos minoritários da indústria, junto com a categoria "children", "gospel", "classical" (música clássica) e"new age", conforme as estatísticas da Nielsen Company (Richard, 2009). Esse resultado apresenta uma situação paradoxal: ao mesmo tempo em que a música eletrônica foi o quarto gênero mais escutado na Last.fm em 2009 (Last.Fm, 2009), correspondeu a um dos menores mercados da indústria no mesmo ano.

Uma das possibilidades para explicar esse fenômeno é que os produtores e os circuitos comerciais de música eletrônica passam por uma produção e distribuição que escapa ao campo de atuação exclusivo da Indústria Fonográfica. Por exemplo, o gênero eletrônico é o que possui mais artistas independentes que disponibilizam suas músicas na Last.fm.
Cabe uma última observação sobre o gênero rock. De acordo com o mesmo relatório da Nielsen Company (Richard, 2009), o rock é o gênero que isoladamente mais vende, tanto por meio de suportes físicos como digitais. Porém, as análises comparativas realizadas nesta pesquisa mostram que o rock não ocupa nenhum dos extremos na tabela apresentada.

Esse gênero combina dois fatores contraditórios que articulados explicam sua posição intermediária na Tabela 2. Mesmo sendo o gênero mais vendido pelas grandes gravadoras - o que diante da proposição sugerida representa um fator que impulsionaria a semelhança entre a classificação nos dois domínios -, o rock é uma categoria musical demasiado ampla para os consumidores, apesar de ser uma importante categoria de classificação para indústria. Para os usuários, o rock representa um estilo de música com ilimitados subgêneros - ou seja, possui uma classificação altamente diferenciada no âmbito das práticas culturais, principalmente por meio de combinações híbridas com outros gêneros, como, por exemplo, pop-rock, hard-rock, punk-rock etc.

Durante a coleta de dados, percebeu-se que o cruzamento destes dois fatores gera um atrito entre duas forças: ao mesmo tempo em que a maior penetração no mercado aproxima a classificação dos usuários da classificação comercial, as fracas fronteiras que definem esse gênero tendem a torná-lo mais diferenciado, com múltiplas subdivisões e associações híbridas com outros gêneros. A soma dessas duas variáveis explica por que o rock localiza-se em uma posição mediana na Tabela 2.

\section{Os princípios comerciais de classificação da música e suas implicações sociais}

A classificação da música popular - tanto aquela utilizada pela indústria musical como as novas categorias criadas pelos usuários na Internet - engendra importantes implicações nas práticas culturais que aparecem e desaparecem no campo social.

O desafio é entender o processo pelo qual as similaridades são percebidas e os gêneros musicais decretados. Baseando-se especialmente no pensamento de DiMaggio (1987).

Para DiMaggio (1987), os procedimentos pelos quais distintos gêneros são criados, inseridos nos hábitos 
do público ou desconstruídos estão diretamente relacionados com dois processos pelos quais os gostos são produzidos: 1) como parte da construção de sentido dos produtos culturais e 2) como mecanismo estruturante das atividades que definem as fronteiras entre os grupos sociais. Ou seja, a classificação social dos gêneros está implicada no processo pelo qual a música é classificada e ao mesmo tempo "classificante".

Esses dois processos sociais se aglutinam no que DiMaggio (1987) chama de Sistema de Classificação Artístico (SCA). Um SCA se refere à forma como as obras e/ou os artistas são divididos tanto na percepção cognitiva e nos hábitos dos consumidores como para as instituições envolvidas no mercado artístico que organizam a oferta e delimitam a produção e distribuição em gêneros separados.

Um Sistema de Classificação Artístico indica os princípios de relações estabelecidas entre os gêneros e também entre os artistas em uma dada coletividade. Dessa forma, um SCA reflete tanto a estrutura de divisão do gosto de uma população como a estrutura da produção e distribuição dos bens culturais (DiMaggio, 1987). Para adaptar a nomenclatura ao enfoque desta pesquisa, a expressão "Sistema de Classificação Artístico" (SCA) é utilizada para referir-se aos sistemas de classificação no domínio específico da música popular.

O Sistema de Classificação Artístico encontrado no AllMusic foi denominado como SCA comercial por representar um sistema amplamente utilizado pela indústria musical. A classificação encontrada na Last.fm chamou-se de SCA social ou não comercial, criada e utilizada pela rede de ouvintes desta plataforma e, portanto, baseada nos usos sociais da música na Internet.

A partir do próprio conceito de SCA, cabe perguntar sobre a função social de um sistema de classificação artístico em categorias de gêneros musicais. Por um lado, a classificação em gêneros permite que os consumidores invistam em conhecimento especializado e, por outro, que os artistas possam alocar seu trabalho em um dado "mercado". Conforme demonstra Becker (1982), os artistas trabalham com base em "áreas familiares" que institucionalizam os "mundos da arte" tanto no nível da oferta como no da demanda, com convenções que tornam a produção possível. Portanto, de acordo com o Becker
(1982), a classificação dos gêneros socializa os custos de "infraestrutura simbólica" da produção artística.

Por outro lado, segundo DiMaggio (1987), os interesses comerciais normalmente reforçam os rituais de classificação dividindo a sociedade em grupos, segmentos ou nichos, o que ajuda a organizar a constituição social dos gêneros. O exemplo mais relevante é o da Indústria Fonográfica com seus estratos de idade/ classe/etnia. A "invenção" de categorias musicais como "adult contemporary","tastemakers","latin music" pelo mercado musical corresponde menos a gêneros musicais do que às estratégias comerciais para a segmentação (ou classificação) dos públicos.

Embora os discursos sob os quais os gêneros são organizados tenham diversas implicações, a dinâmica que se ressalta nos "rituais de classificação" é a relação que se estabelece entre os fatores socioestruturais que influenciam a demanda cultural, a maneira como essa demanda é organizada e a forma como os bens culturais são classificados em gêneros dotados de sentido social.

Os rituais de classificação variam de acordo com a intensidade na qual as fronteiras são socialmente defendidas tanto no nível da produção artística como no nível do consumo. Para que as fronteiras entre os gêneros sejam defendidas no campo social é necessário que sejam amplamente entendidas. O aumento da oferta e da disponibilidade de produtos culturais diversificados estimula o processo de deslocamento social intergrupos em fronteiras mais flexíveis e impulsiona a demanda social por formas culturais diferenciadas (DiMaggio, 1987).

Diante dessa perspectiva e ao considerar o conjunto de proposições organizadas por DiMaggio (1987), pode parecer implícito que os SCA refletem perfeitamente as divisões existentes no campo social. Entretanto, esta correspondência não ocorre de forma coerente devido ao fato de que a organização das obras musicais na sociedade é mediada por sistemas de classificação comercial.

Contraditoriamente, os Sistemas de Classificação Artísticos comerciais estão subordinados, em certo grau, aos próprios processos de ritualização dos gêneros no nível social (DiMaggio, 1987). As Indústrias Culturais tentam reproduzir e estabilizar as fronteiras "previamente" existentes entre os grupos sociais a fim de rentabilizar 
sua atuação no mercado e diminuir os riscos de seu negócio.

Portanto, os princípios comerciais de categorização da cultura diferem dos sociais em uma forma fundamental: os rituais de classificação respondem às demandas socioestruturais dos consumidores no nível dos usos sociais, enquanto a classificação comercial reflete a estrutura da produção e distribuição das Indústrias Culturais.

A eficácia dos SCA comerciais depende de sua correspondência com os circuitos sociais de uso e paralelamente com as lógicas do seu sistema de produção e distribuição. Por isso, o mercado musical adapta, na medida do possível, a atualização de seu sistema de classificação de música de acordo com os fluxos sociais - desde que esse processo esteja articulado com os interesses e as limitações da própria lógica industrial.

Quando essa articulação não acontece, a classificação comercial pode ser usurpada por grupos sociais para seus propósitos de uso, como acontece com a lógica de classificação social presente na Last.fm que corrompe alguns princípios do mercado tradicional. Por outro lado, as Indústrias Culturais constantemente usurpam, desativam e difundem rituais de classificação opostos ou contraditórios aos usos sociais, de acordo com seus fins mercantis (Hebdige, 1979).

Os Sistemas de Classificação Artísticos comerciais correspondem às fronteiras impostas por empresas que buscam o lucro em uma economia de mercado. Os SCA comerciais emergem por meio de processo de identificação e segmentação dos mercados com base nas estratégias de maximização das receitas das empresas implicadas. Com a ajuda de publicidade e canais especializados de difusão da informação que servem ao mercado, as Indústrias Culturais criam diferentes graus de percepção e acesso aos gêneros entre distintos segmentos do público consumidor (DiMaggio, 1977). Os grupos que possuem status social tentam monopolizar os bens simbólicos para intensificar seus rituais de inclusão e diferenciação (Bernstein, 1973). E como demonstra DiMaggio (1987), sob algumas circunstâncias, a classificação comercial reforça tais rituais de classificação.

Ao mesmo tempo, frequentemente os sistemas de classificação comerciais tentam romper com os rituais de classificação, ou seja, com o cultivo das fronteiras entre os grupos de gosto. Como os produtores comerciais buscam grandes mercados e economia de escala, quanto menos diferenciados os gêneros, mais amplos os seus mercados e consequentemente mais lucrativos.

Os produtores comerciais pretendem expandir os mercados ao máximo, mesmo sob o risco de reduzir o valor ritual e social dos produtos que vendem. Normalmente, as obras que atingem porcentagens consideráveis de venda geram mais lucro do que aquelas que atraem vários, porém pequenos, grupos de aficionados. Dessa forma, a discrepância entre o valor comercial e o valor simbólico cria uma oposição entre os princípios da classificação ritualizada socialmente e os critérios comerciais na competição entre mercado e status cultural (Weber, 1968; Peterson, 1978; Bourdieu, 1985; DiMaggio, 1987).

O efeito dessa estratégia mercadológica é paradoxal: na medida em que aumenta a dependência pelo retorno dos investimentos baseados na economia de escala, as empresas reduzem a heterogeneidade de seus repertórios. A dialética entre homogeneização da oferta e massificação do consumo deprecia cada vez mais o valor social dos gostos, incluindo a desvalorização do status de determinados gêneros (DiMaggio; Stenberg, 1985).

Ao mesmo tempo, as Indústrias Culturais fragmentam as audiências em nichos de consumidores com perfis sociodemográficos aproximados para tentar reduzir os riscos de rejeição dos seus produtos lançados no mercado. E assim as grandes empresas no ramo da música reinventam mecanismos de estratificação social não mais baseados nas diferenças de status e gosto, e sim no poder de compra dos diferentes segmentos.

\section{Conclusão}

Em áreas competitivas como o campo da música popular, o mercado - especialmente com o amadurecimento das Indústrias Culturais -, tem funcionado como um campo de força magnética em torno do qual os gêneros musicais são consolidados. Desse modo, a percepção cognitiva dos gêneros ocorre por meio de criação, difusão e interpretação das informações do "mercado" pelos grupos sociais que propagam seu valor e reproduzem sua lógica no campo das práticas. Portanto, um sistema 
de classificação artístico tende a refletir tanto a estrutura da produção e distribuição dos bens simbólicos como a organização social dos gostos culturais.

A indústria da música opera determinados sistemas de classificação artísticos com o intuito de organizar a oferta e a demanda de acordo com as conveniências e coerências de produção e distribuição e assim tenta diminuir os riscos de sua atuação comercial. Entretanto, a popularização da Internet e seus efeitos de facilidade de acesso, gratuidade e banalização do status cultural geram pressões em direção à desmistificação e ao enfraquecimento dos rituais de classificação que fazem circular categorias comerciais estigmatizadas. No âmbito da filtragem e classificação colaborativa na Rede, vê-se surgir critérios e categorias que organizam a oferta musical de acordo com as práticas dos próprios usuários.

Os resultados encontrados nesta pesquisa demonstram que, na medida em que os usuários passam a classificar e a representar a informação de acordo com as suas percepções, afecções e os seus hábitos musicais, a classificação das obras escapa aos padrões comerciais que orientam os processos cognitivos e os usos sociais da música. Foi encontrada uma forte discrepância entre os usos sociais e os critérios comerciais de classificação, o que pode apontar para uma crise dos modelos de representação da música moldados e controlados pela indústria fonográfica.

Por isso, quando se compara os critérios de semelhança utilizados pela indústria para associar artistas e organizar o mercado musical com as classificações e os usos efetivos dos usuários, a compatibilidade é mínima. Ou seja, os artistas considerados pela indústria como parecidos, pertencentes a um mesmo "segmento de mercado" diferem significativamente daqueles associados pelos usuários e realmente utilizados por um mesmo público. A análise dos dados desta pesquisa indi- ca que a percepção e os gostos dos ouvintes não correspondem aos critérios comerciais de "nicho", porém esta proposição deve ser testada empiricamente em outras pesquisas que utilizem diferentes amostras e métodos.

Considerou-se que as condições sociais para a criação de novas formas de organização social da cultura são dadas a partir do momento em que os próprios usuários podem interferir coletivamente e de forma horizontal em sua classificação e mediação. As plataformas colaborativas oferecem possibilidades de reapropriação dos dispositivos de construção das subjetividades cuja autonomia se exerce pelo poder de classificar para si e de não se deixar ser classificado por outros.

Cabe ponderar que a grande massa de usuários da Internet ainda encontra-se em um momento de aprendizado e desenvolvimento de familiaridade diante das possibilidades oferecidas pelas tecnologias de filtragem colaborativa, porém seu uso começa a ocupar um lugar privilegiado nos seus hábitos cotidianos - o que pode encorajar ou inibir a dominação de determinados sistemas de classificação artísticos sobre outros.

Portanto, este trabalho aponta para uma agenda de pesquisa nas Ciências Sociais - especialmente para a Sociologia da cultura e a Comunicação Social -, que considere as implicações sociais dos sistemas de classificação colaborativos nos contextos online de produção, mediação, difusão, consumo e uso de bens culturais. É importante que futuras pesquisas contribuam para identificar o lugar que efetivamente podem ocupar os usos e usuários diante da dialética entre as determinações materiais e simbólicas. Esta complexa relação de interdependência age sobre as estruturas sociais e psicológicas dos agentes implicados e define as lógicas sociais que podem se tornar dominantes no campo cultural.

\section{Referências}

ALLMUSIC LDT. Coverage statistics. 2009. Available from: $<$ http://www.allmusic.com/cg/amg.dll?p=amg\&sql=32: amg/info_pages/a_about_cover_stats.html>. Cited: Mar. 22, 2009.

BECKER, H.S. Art worlds. Berkeley: University of California Press, 1982.

BERNSTEIN, B. Class, codes and control. Boston: Routledge, 1973.

BOURDIEU, P. The field of cultural production, or: the economic world reversed. Poetics, n.12, p.311-356, 1985.

DIMAGGIO, P.I. Market structures, the creative process, and popular culture. Journal of Popular Culture, v.11, p.436-452, 1977.

DIMAGGIO,P.Classification in art. American Sociological Review, v.52, p.440-455, 1987. 
DIMAGGIO, P.; STENBERG, K. Conforming and diversity in the american regional stage. In: BALFE, J.; WYSZOMIRSKI, M. Art, ideology and politics. New York: Praeger, 1985. p.116-140.

HEBDIGE, D. Subculture: the meaning of style. London: Methuen, 1979.

HENNING, V.; REICHELD, J. Mendeley: a last.fm for research? In: IEEE INTERNATIONAL CONFERENCE ON E-SCIENCE, 4., 2008, Indianapolis, USA. Procceedings... Indianapolis: Indiana University, 2008.

LAST. FM. Last.fm: radio announcement. 2009. Available from: <http://blog.last.fm/2009/03/24/lastfm-radio-announcement>. Cited: Mar. 24, 2009.
MILLER, F.; STIKSEL, M.; JONES, R. Last.fm in numbers. London: CBS Interative, 2008.

RICHARD, J. State of the industry 2007-2008: Last HQ. 2009. Available from: <http://www.narm.com/Research/Nielsen08. pdf>. Cited: Dec. 30, 2008.

PETERSON, R.A. The production of cultural change: the case of contemporany country music. Social Research, v.45, p.292-314, 1978

THOMPSON, A.E. Playing tag: an analysis of vocabulary patterns and relationships within a popular music folksonomy. Chapel Hill: University of North Carolina Press, 2008.

WEBER, M. Economy and society. New York: Bedminster, 1968. 